\title{
Dark matter as a localized scalar in the extra dimension
}

\author{
E. O. Iltan * \\ Physics Department, Middle East Technical University \\ Ankara, Turkey
}

\begin{abstract}
We consider a standard model singlet which is accessible to a single extra dimension and its zero mode is localized with Gaussian profile around a point different than the origin. This zero mode scalar is a possible candidate for the dark matter and its annihilation rate is sensitive to the compactification radius of the extra dimension, the localization width and the position. For the case of non resonant annihilation, we estimated the dark matter scalar location around a point, at a distance $\sim 3 \times$ localization width from the origin, by using the annihilation rate which is based on the current relic density.
\end{abstract}

${ }^{*}$ E-mail address: eiltan@newton.physics.metu.edu.tr 
The amount of matter required in the universe is considerably greater than the visible one and there is a need to construct a theoretical background in order to explain the excess invisible matter, called as dark matter (DM). The galactic rotation curves [1] and galaxies orbital velocities [2], the cosmic microwave background anisotrophy [3], observations of type Ia supernova [4] indicate that almost $23 \%$ of present Universe [4, 5, 6] is composed of cold (non relativistic) DM, however, its nature is not known. In the literature, there are various attempts to solve the DM problem; the DM candidates in supersymmetric models [7], in the universal extra dimension (UED) models [8, 9, 10, split UED models [11, 12, 13, Private Higgs model [14], Inert doublet model [15]-[22], Little Higgs model [23], Heavy Higgs model [24]. Among the possible candidates the Weakly Interacting Massive Particles (WIMPs) that interact only through weak and gravitational interactions and have masses in the range $10 \mathrm{GeV}$ - a few $\mathrm{TeV}$ reached great interest since the current relic density could be explained by thermal freeze-out of their pair annihilation (see for example [25, 26] for further discussion).

In the present work we consider a scalar SM singlet $\phi_{S}$ which is accessible to a single extra dimension and all SM particles live on the 4 dimensional brant 1 . After the compactification of the extra dimension, denoted by $y$, on an orbifold $S^{1}$ with radius $R$ the KK modes of the SM singlet scalar appear. At this stage, we assume that the zero mode scalar is localized2 (see for example [35] for a special localization mechanism for a scalar in the extra dimension) at the point $y_{L}$ in the extra dimension, with the Gaussian profile as

$$
\phi_{S}^{(0)}=f_{L}(y) S(x)
$$

where the function $f_{L}(y)$ reads

$$
f_{L}(y)=A_{L} e^{-\beta\left(y-y_{L}\right)^{2}}
$$

The normalization constant $A_{L}$ is obtained by using the integral

$$
\int_{-\pi R}^{\pi R} f_{L}^{2}(y) d y=1
$$

and one gets

$$
A_{L}=\frac{2(\beta)^{1 / 4}}{\left.(2 \pi)^{1 / 4} \sqrt{\operatorname{Erf}\left[\sqrt{2 \beta}\left(\pi R+y_{L}\right)\right]+\operatorname{Erf}\left[\sqrt{2 \beta}\left(\pi R-y_{L}\right)\right.}\right]} .
$$

\footnotetext{
${ }^{1}$ See [27] and [28, 29, 30, 31, 32, 33, 34] for an additional scalar SM singlet field $\phi_{S}$ in 4 dimensions, called as darkon

${ }^{2}$ We consider that the mechanism for the localization is unknown.
} 
Here $\beta, \beta=1 / \sigma^{2}$, is the parameter which adjusts the localization amount of $\phi_{S}^{(0)}$ and $\sigma$ is the Gaussian width of $\phi_{S}^{(0)}$ in the extra dimension. The function $\operatorname{Er} f[y]$ is the error function, which is defined as

$$
\operatorname{Erf}[y]=\frac{2}{\sqrt{\pi}} \int_{0}^{y} e^{-t^{2}} d t
$$

Furthermore, we assume that the lagrangian of the scalar SM singlet has the discrete $Z_{2}$ symmetry, $\phi_{S}^{(0)} \rightarrow-\phi_{S}^{(0)}$ in order to ensure its stability and has the interaction term in a single extra dimension 3

$$
\mathcal{L}_{\text {Int }}=\left.\lambda_{5 S} \phi_{S}^{(0) 2}\right|_{y=0}\left(\Phi_{1}^{\dagger} \Phi_{1}\right)
$$

where $\Phi_{1}$ is the SM Higgs field

$$
\phi_{1}=\frac{1}{\sqrt{2}}\left[\left(\begin{array}{c}
0 \\
v+H^{0}
\end{array}\right)+\left(\begin{array}{c}
\sqrt{2} \chi^{+} \\
i \chi^{0}
\end{array}\right)\right]
$$

with the vacuum expectation value

$$
<\phi_{1}>=\frac{1}{\sqrt{2}}\left(\begin{array}{l}
0 \\
v
\end{array}\right)
$$

Here we consider that the SM singlet scalar $\phi_{S}$ has no vacuum expectation value and, with the discrete $Z_{2}$ symmetry above, it is guaranteed that the scalar field $S$ has no SM decay products. After the electroweak symmetry breaking, they disappear by pair annihilation with the help of the exchange particle which is the SM Higgs boson $H^{0}$ in this case. Therefore, the zero mode scalar is a possible candidate for DM and we will study the effects of compactification radius, the zero mode scalar localization width and its localization position on the annihilation cross section.

The annihilation process $S S \rightarrow H^{0} \rightarrow X_{S M}$ results in the total averaging annihilation rate of $S S$

$$
<\sigma v_{r}>=\frac{4 \lambda_{S}^{2} v^{2}}{m_{S}} \frac{1}{\left(4 m_{S}^{2}-m_{H^{0}}^{2}\right)^{2}+m_{H^{0}}^{2} \Gamma_{H^{0}}^{2}} \Gamma\left(\tilde{h} \rightarrow X_{S M}\right)
$$

where $\Gamma\left(\tilde{h} \rightarrow X_{S M}\right)=\sum_{i} \Gamma\left(\tilde{h} \rightarrow X_{i S M}\right)$ with virtual Higgs $\tilde{h}$ having mass $2 m_{S}$ (see [36, 37]) and $v_{r}=\frac{2 p_{C M}}{m_{S}}$ is the average relative speed of two zero mode scalars (see for example [34]). In eq.(9) the effective coupling $\lambda_{S}$ reads

$$
\lambda_{S}=\lambda_{5 S} A_{L}^{2} e^{-2 \beta y_{L}^{2}}
$$

\footnotetext{
${ }^{3}$ Here an ad hoc non zero mass term for the zero mode singlet $\phi_{S}^{(0)}$ is considered and it depends on the localization mechanism which is unknown in our case. Notice that the electroweak symmetry breaking also leads to a contribution to the tree level mass due to the interaction term (see eq.(6) ).
} 
Here, we parametrize $\sigma$ and $y_{L}$ as $\sigma=\rho R, y_{L}=\alpha \sigma$ where $\rho$ and $\alpha$ are dimensionless parameters and, for the effective coupling $\lambda_{S}$, we get

$$
\lambda_{S}=\frac{4 e^{-2 \alpha^{2}}}{\sqrt{2 \pi} \rho R\left(\operatorname{Erf}\left[\sqrt{2}\left(\frac{\pi}{\rho}+\alpha\right)\right]+\operatorname{Erf}\left[\sqrt{2}\left(\frac{\pi}{\rho}-\alpha\right)\right]\right)},
$$

with the choice $\lambda_{5 S}=1.0 \mathrm{GeV}^{-1}$. Therefore, in this scenario, the strength of tree level interaction of the scalar DM pair with the SM Higgs boson is regulated by the scalar DM localization point, its localization width and the compactification radius of the extra dimension. In order to estimate these parameters one needs a restriction for the annihilation cross section $\left\langle\sigma v_{r}>\right.$. The present DM abundance by the WMAP collaboration [38] is

$$
\Omega h^{2}=0.111 \pm 0.018
$$

and the annihilation cross section is inversely proportional to the relic density as

$$
\Omega h^{2}=\frac{x_{f} 10^{-11} \mathrm{GeV}^{-2}}{<\sigma v_{r}>},
$$

where $x_{f} \sim 25$ [5, 11, 34, 39, 40]. Finally, eqs.(12) and (13) lead to the bounds for the annihilation cross section,

$$
<\sigma v_{r}>=0.8 \pm 0.1 p b
$$

in the case that s-wave annihilation is dominant (see [41] for details.).

\section{Discussion}

In the present work we introduce a scalar field, which is a SM singlet, with vanishing vacuum expectation value and consider that its zero mode localized with Gaussian profile around a point away form the origin, in the extra dimension. The interaction Lagrangian (see eq. (6) ) results in that this field interacts with the SM Higgs $H^{0}$ and the tree level interaction $S S H^{0}$ arises with the effective coupling $\lambda_{S}$ (eq.(11) ) after the elecroweak symmetry breaking. This coupling drives the annihilation cross section which should be compatible with the present observed DM relic density (eq.(12)). The free parameters of the model used are the Higgs mass $m_{H^{0}}$, the zero mode scalar mass $m_{S}$, the compactification radius $R$ of the extra dimension, the localization position $y_{L}$ of the zero mode scalar and its localization width $\sigma$. Here, we take Higgs mass around $110-120 \mathrm{GeV}$ and use the range $10-80 \mathrm{GeV}$ for the zero mode scalar mass. Furthermore, we respect the prediction of the present DM abundance and use the central value 
of $\left\langle\sigma v_{r}\right\rangle=0.8 p b$ in order to predict the localization position of the DM with respect to the compactification radius $R$ and the DM mass $m_{S}$. In the numerical calculations we take the compactification radius $R$ in the range $0.00001 \mathrm{GeV}^{-1} \leq R \leq 0.001 \mathrm{GeV}^{-1}$. In the range of free parameters we choose, the coupling $\lambda_{S}$ obeys $\lambda_{S}<1.0$, which is necessary for perturbative calculations. Notice that the direct detection experiments ensure an upper limit (see [42] for the current upper limit) for the WIMP-nucleon cross section 4 . The parameter set we used leads to the cross section for proton target at the order of magnitude of $10^{-8}-10^{-7} p b$ which is almost one order less than the current limit. This prediction shows that the considered parameter set, which respects the bounds of the annihilation cross section, is not rule out by the results of direct detection that might be improved with the forthcoming experiments.

In Fig.1 we plot the compactification radius $R$ dependence of $\alpha$ for $m_{H^{0}}=110 \mathrm{GeV}$. Here the upper-intermediate-lower solid (long dashed; dashed) line represents $\alpha$ for $m_{S}=50-80-$ $10 \mathrm{GeV}, \rho=0.001(0.01 ; 0.1)$. It is observed that the parameter $\alpha$, which measures the distance of the localization point of the DM scalar from the origin in the extra dimension, is in the range of $2.4 \sigma-3.3 \sigma$, for the chosen interval of the free parameters. For $m_{S}=50 \mathrm{GeV}$, the mass of the scalar which is near to the resonant annihilation, the interaction coupling $\lambda_{S}$ should be regulated to a small value in order to reach the appropriate annihilation cross section which agrees with the current relic density. This is the case that the DM scalar is localized far from the origin in order to weaken the interaction with the Higgs particle and, therefore, the parameter $\alpha$ reaches to relatively greater values. In the case of the DM with the mass value far from the resonant annihilation, the heavier DM has a weak cross section compared to the lighter one and, in order to satisfy the observed relic abundance, it should be pulled to the appropriate value by choosing the weaker coupling, i.e. relatively larger $\alpha$. This figure shows that $\alpha$ is sensitive to the parameter $\rho$, which fixes the width of the localization, and to the compactification radius $R$. With the increasing values of $\rho$ and $R \alpha$ decreases 5 .

Fig,2 represents the DM scalar mass $m_{S}$ dependence of $\alpha$ for $m_{H^{0}}=110 \mathrm{GeV}$. Here the upper-lower solid (long dashed; dashed) line represents $\alpha$ for $R=0.001-0.005 \mathrm{GeV}^{-1}, \rho=$ $0.001(0.01 ; 0.1)$. We observe that the parameter $\alpha$ reaches to the largest value $\sim 3.6 \sigma$ in the case of resonant annihilation and decreases when the DM mass becomes far from the resonance mass, $m_{S}=55 \mathrm{GeV}$. Near the resonant case $\alpha$ is sensitive the DM scalar mass and this sensitivity

\footnotetext{
${ }^{4}$ The spin independent cross section can be given as $\sigma=\frac{f^{2} m_{N}^{2} m_{S N}^{2}}{4 \pi}\left(\frac{\lambda_{S}}{m_{S} m_{H^{0}}^{2}}\right)^{2}$ where $m_{N}\left(m_{S N}\right)$ is the nucleon mass (reduced mass of $S$ and nucleon) and the coupling $f$ reads $f \sim 0.3$ (see for example [16, 43]).

${ }^{5}$ Notice that the width of the localization which is parameterized by $\rho$ is chosen at least one order less than the compactification radius $R$
} 
becomes weak when the mass $m_{S}$ is far from $55 \mathrm{GeV}$, in the range of $m_{S}$ considered. It is seen that, for the large mass values, $m_{S} \geq 80 \mathrm{GeV}$, this sensitivity becomes strong and $\alpha$ increases, since the annihilation cross section enhances and it should be suppressed by appropriate weak coupling which is regulated by the parameter $\alpha$.

In Fig 3 we present the DM scalar mass $m_{S}$ dependence of $\alpha$ for $R=0.001 \mathrm{GeV}^{-1}$. Here the left-right solid (long dashed; dashed) line represents $\alpha$ for $m_{H^{0}}=110-120 \mathrm{GeV}, \rho=$ $0.001(0.01 ; 0.1)$. Here two maximum values of $\alpha$ at different $m_{S}$ are due to two different resonant annihilations, namely the annihilations for $m_{S}=55 \mathrm{GeV}$ and $m_{S}=60 \mathrm{GeV}$. This figure shows that $\alpha$ is greater (smaller) almost for $m_{S}>57 \mathrm{GeV}\left(m_{S}<57 \mathrm{GeV}\right)$ in the case of $m_{H^{0}}=120 \mathrm{GeV}$ compared to the case of $m_{H^{0}}=110 \mathrm{GeV}$. With the increasing values of the Higgs mass, the localization point of the scalar DM in the extra dimension goes far from (comes near to) the origin if the scalar DM mass $m_{S}$ is greater (less) than the resonant annihilation mass.

As a summary, we consider that the additional scalar field is accessible to a single extra dimension and its zero mode is localized. With the ad hoc symmetry in the Lagrangian assumed this zero mode becomes stable and annihilates to the SM Higgs particles and, therefore, it is a candidate for a scalar DM. In this scenario, we estimate the position of the localization point of the scalar DM in the extra dimension by respecting the current relic density. We observed that the localization point of the DM scalar places at $2.4 \sigma-3.4 \sigma$ distance from the origin in the extra dimension, for the chosen interval of the free parameters. In the case of the resonant annihilation this distance reaches to $3.6 \sigma$. Furthermore we see that this distance decreases with the increasing values of localization width parameter $\rho$ and compactification radius $R$ and it is sensitive to the SM Higgs boson mass. Hopefully, the observation of the SM Higgs boson in the future experiments at LHC will provide a considerable information about the nature of the DM and the possible mechanism which drives the DM-SM Higgs annihilation. 


\section{References}

[1] A. Borriello and P. Salucci, Mon. Not. Roy. Astron. Soc. 323, 285 (2001).

[2] F. Zwicky, Helv. Phys. Acta. 6, 110 (1993).

[3] D. N. Spergel et.al (WAMP Collaboration), Astrophys. J. Suppl. Ser. 170377 (2007).

[4] E. Komatsu et al., Astrophys. J. Suppl. Ser. 180, 330 (2009).

[5] G. Bertone, D. Hooper and J. Silk, Phys. Rept. 405, 279 (2005).

[6] G. Jungman, M. Kamionkowski and K. Griest, Phys. Rept. 267, 195 (1996).

[7] M. Asano, S. Matsumoto, M. Senami, H. Sugiyama, Phys. Lett. B663, 330 (2008).

[8] T. Appelquist, H. C. Cheng and B. A. Dobrescu, Phys. Rev. D64, 035002 (2001).

[9] H. C. Cheng, K. T. Matchev and M. Schmaltz, Phys. Rev. D66, 056006 (2002).

[10] S. Matsumoto, J. Sato, M. Senami, M. Yamanaka, hep-ph/0903.3255.

[11] G. Servant and T. M. P. Tait, Nucl. Phys. B650, 391 (2003).

[12] S. C. Park and J. Shu, Phys. Rev. D79, 091702(R) (2009).

[13] C. R. Chen, M. M. Nojiri, S. C. Park, J. Shu, M. Takeuchi, hep-ph/0903.1971.

[14] C.B. Jackson, hep-ph/0804.3792.

[15] E. Ma, Phys. Rev. D73, 077301 (2006).

[16] R. Barbieri, L. J. Hall, and V. S. Rychkov, Phys. Rev. D74, 015007 (2006).

[17] M. Cirelli, N. Fornengo, and A. Strumia, Nucl. Phys. B753, 178 (2006).

[18] N. G. Deshpande and E. Ma, Phys. Rev D18, 2574 (1978).

[19] J. A. Casas, J. R. Espinosa, and I. Hidalgo, Nucl. Phys. B777, 226 (2007).

[20] L. L. Honorez, E. Nezri, J. F. Oliver, M. H. G. Tytgat, JCAP 0702, 028 (2007).

[21] S. Andreas, T. Hambye, M. H.G. Tytgat, JCAP 0810, 034 (2008).

[22] X. Calmet and J. F. Oliver, Europhys. Lett. B77, 51002 (2007). 
[23] Y. Bai Phys.Lett. B666, 332 (2008).

[24] D. Majumdar and A. Ghosal, Mod. Phys. Lett. A23, 2011 (2008).

[25] F. D. Eramo Phys. Rev. D76, 083522 (2007).

[26] W. L. Guo, X. Zhang, Phys. Rev. D79, 115023 (2009).

[27] V. Silveira and A. Zee, Phys. Lett. B161, 136 (1985).

[28] C. P. Burgess, M. Pospelov, T. ter Veldhuis, Nucl. Phys. B619, 709 (2001).

[29] D. E. Holz and A. Zee, Phys. Lett. B517, 239 (2001).

[30] J. McDonald, Phys. Rev. D50, 3637 (1994).

[31] B. Patt and F. Wilczek (2006), hep-ph/0605188.

[32] O. Bertolami, R. Rosenfeld, Int. J. Mod. Phys. A23, 4817 (2008).

[33] H. Davoudiasl, R. Kitano, T. Li, and H. Murayama, Phys. Lett. B609, 117 (2005).

[34] X. G. He, T. Li, X. Q. Li, and H.-C. Tsai, Mod. Phys. Lett. A22, 117 (2005).

[35] E. O. Iltan, Eur. Phys. J. C51, 689 (2007).

[36] C. Bird, P. Jackson, R. Kowalewski and M. Pospelov, Phys. Rev. Lett. 93, 201803 (2004).

[37] C. Bird, R. Kowalewski and M. Pospelov, Mod. Phys. Lett. A21, 457 (2006).

[38] D. N. Spergel et.al (WAMP Collaboration), Astrophys. J. Suppl. Ser. 148175 (2003).

[39] S. Gopalakrishna, S. Gopalakrishna, A. de Gouvea, W. Porod, JCAP 0605, 005 (2006).

[40] S. Gopalakrishna, S. J. Lee, J. D. Wells, hep-ph/0904.2007

[41] E. W. Kolb and M. S. Turner, The Early Universe (Addison- Wesley, Reading, MA, 1990).

[42] D. S. Akerib et.al. [CDMS Collaboration], Phys. Rev. Lett. 96, 011302 (2006).

[43] A. Pierce, J. Thaler, JHEP 0708, 026 (2007). 


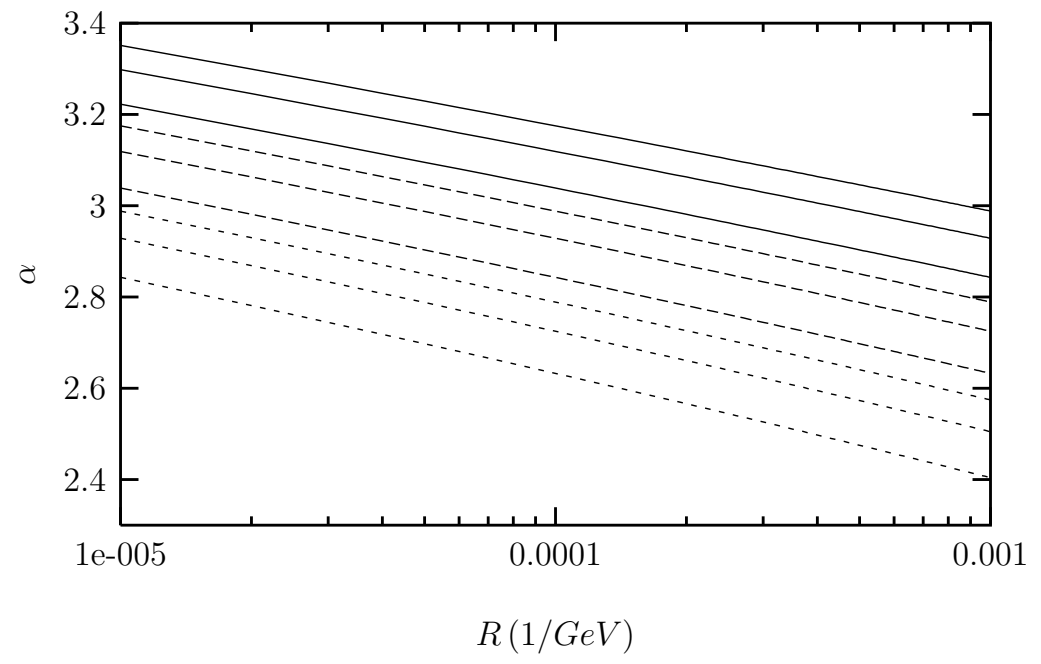

Figure 1: $\alpha$ as a function of $R$ for $m_{H^{0}}=110 \mathrm{GeV}$. Here the upper-intermediate-lower solid (long dashed; dashed) line represents $\alpha$ for $m_{S}=50-80-10 \mathrm{GeV}, \rho=0.001(0.01 ; 0.1)$. 


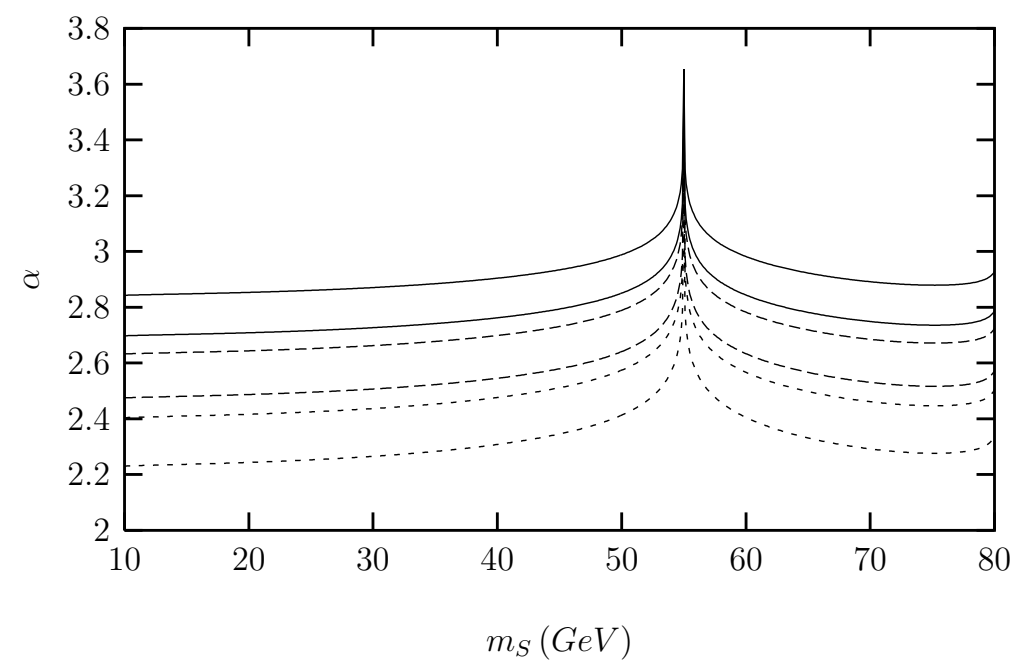

Figure 2: $\alpha$ as a function of $m_{S}$ for $m_{H^{0}}=110 \mathrm{GeV}$. Here the upper-lower solid (long dashed; dashed) line represents $\alpha$ for $R=0.001-0.005 \mathrm{GeV}^{-1}, \rho=0.001(0.01 ; 0.1)$.

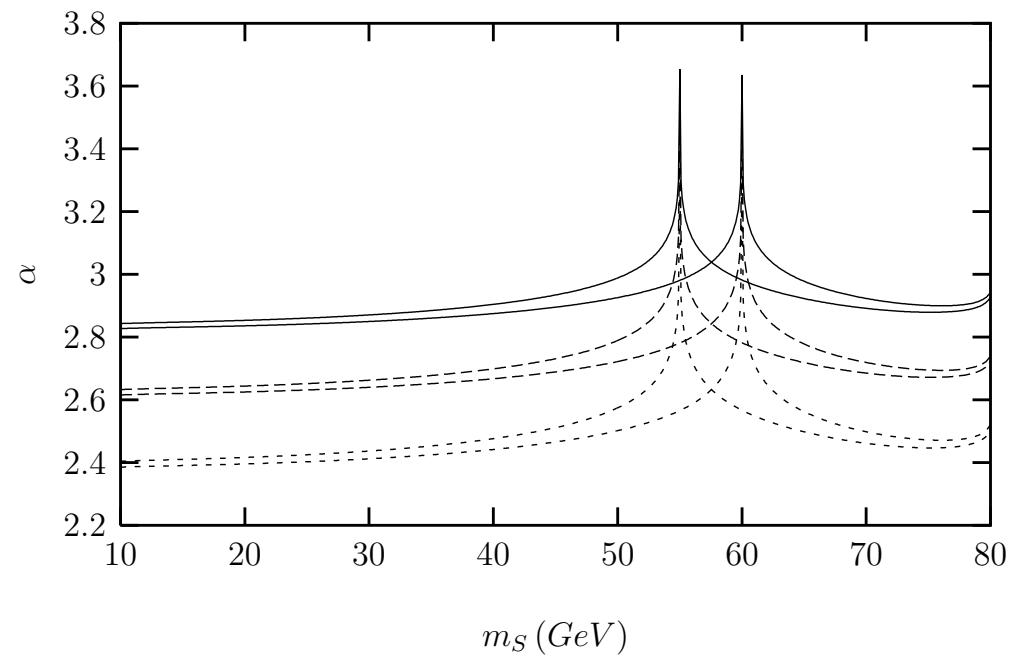

Figure 3: $\alpha$ as a function of $m_{S}$ for $R=0.001 \mathrm{GeV}^{-1}$. Here the left-right solid (long dashed; dashed) line represents $\alpha$ for $m_{H^{0}}=110-120 \mathrm{GeV}, \rho=0.001(0.01 ; 0.1)$. 